Check for updates

Cite this: Chem. Sci., 2019, 10, 8348

๑ All publication charges for this article have been paid for by the Royal Society of Chemistry

\title{
Rational design of a multifunctional molecular dye for dual-modal NIR-II/photoacoustic imaging and photothermal therapy $\dagger$
}

\author{
Ruiping Zhang, $\dot{\dagger}^{\text {ab }}$ Yuling Xu, $\dot{\dagger}^{\mathrm{a}}$ Yi Zhang, $\dot{\hbar}^{\mathrm{a}}$ Hyeong Seok Kim, $\dot{\hbar}^{\mathrm{d}}$ Amit Sharma, \\ Jing Gao, ${ }^{c}$ Guangfu Yang, ${ }^{a}$ Jong Seung Kim (D) *d and Yao Sun (D) *a
}

\begin{abstract}
Small-molecule based multifunctional probes play significant roles in biomedical science and possess high clinical translational ability. However, the preparation of these promising probes without complicated synthetic procedures remains a challenging task. Herein, we rationally designed a high-performance DD-A-DD scaffold molecular dye (SYL) with an intrinsic multifunctional ability and then incorporated it into DSPE-mPEG5000 to facilely construct biocompatible NIR-II fluorescent and photoacoustic (PA) dual-modal theranostic nanoprobes (SYL NPs) ( 120 nm). In vivo studies confirmed that SYL NPS exhibited bright NIR-II fluorescence and PA signals in the tumor region with a promising signal to background ratio (S/B). Meanwhile, SYL NPs demonstrated significantly inhibited tumor growth under laser irradiation with no noticeable side effects. These promising results highlighted SYL NPS as a potential theranostic platform for cancer diagnosis (NIR-II region) and therapy.
\end{abstract}

Received 16th July 2019

Accepted 23rd July 2019

DOI: $10.1039 / c 9 s c 03504 d$

rsc.li/chemical-science

which have high significance and direct impact on the field of

\section{Introduction}

Multifunctional fluorescent probes including multimodal imaging and theranostic probes have evolved into fast-growing research tools with goals of multidimensional visualization of early events in cancer diagnostics and assessment of therapeutic efficacy in vivo. ${ }^{1-8}$ Considering the translational potential of fluorescent probes in preclinical and clinical practice, smallmolecule multifunctional probes remain as exceptional candidates owing to their high biocompatibility, rapid metabolism, and well-defined architectures. ${ }^{9-13}$ However, the widespread applications of such promising probes in biomedicine are heavily hampered as introducing different functional molecules into the molecular platform usually involves abundant and complex chemistry including multiple synthetic steps, tedious chromatographic separations, and issues of chemo-selectivity. ${ }^{14-17}$ Hence, continuous efforts should be made to design simple fluorophores with intrinsic multifunctional ability,

\footnotetext{
${ }^{a}$ Key Laboratory of Pesticides and Chemical Biology, Ministry of Education, International Joint Research Center for Intelligent Biosensor Technology and Health, Center of Chemical Biology, College of Chemistry, Central China Normal University, Wuhan 430079, China. E-mail: sunyaogbasp@mail.ccnu.edu.cn

${ }^{b}$ Affiliated Da Yi Hospital of Shanxi Medical University, Taiyuan 020001, China

'Jiangsu Key Laboratory of Medical Optics, Suzhou Institute of Biomedical Engineering and Technology, Chinese Academy of Sciences, Suzhou215163, China

${ }^{d}$ Department of Chemistry, Korea University, Seoul 02841, Korea

$\dagger$ Electronic supplementary information (ESI) available. See DOI: $10.1039 / \mathrm{c} 9 \mathrm{sc} 03504 \mathrm{~d}$

$\ddagger$ These authors contributed equally to this work.
}

\section{fluorescence imaging and biomedicine. ${ }^{18,19}$}

Over the past several years, fluorescence based imaging in the second near-infrared channel (NIR-II, 1000-1700 nm) has gained attention for its prominent merits and surpasses the conventional visible/NIR-I channels (700-900 nm) in terms of lower tissue scattering and minimal auto-fluorescence, thus leading to significant advances in imaging qualities including image depth and spatiotemporal resolution. ${ }^{20-38}$ More recently, based on a benzobisthiadiazole (BBTD) acceptor, a series of small molecular dyes with a donor-acceptor-donor (D-A-D) architecture have been developed. The reduced energy gap between the hybridized highest occupied molecular orbital (HOMO) and lowest unoccupied molecular orbital (LUMO) levels in these molecular systems shifted the fluorescence emission spectrum from the NIR-I region to the NIR-II channel. ${ }^{39-44}$ Benefiting from their excellent photo-stability and fluorescence features, diverse biological practices such as vascular/lymphatic and tumor fluorescence imaging with a high signal to background ratio (S/B) as well as precise fluorescence image-guided surgery indeed bring good news for biomedical applications in the NIR-II channel. ${ }^{45-60}$ Despite these excellent fluorescence imaging results, the potential utilization of these molecular dyes for multimodal imaging or even cancer theranostics is rarely explored.

In this study, an intrinsic multifunctional and high-performance dye (SYL) based on a DD-A-DD scaffold was rationally designed and facilely prepared (Fig. 1). The designed probe is expected to present the following advantages. Compared with most existing D-A-D based molecular dyes, SYL could achieve better fluorescence performance. This is due to the enhanced 


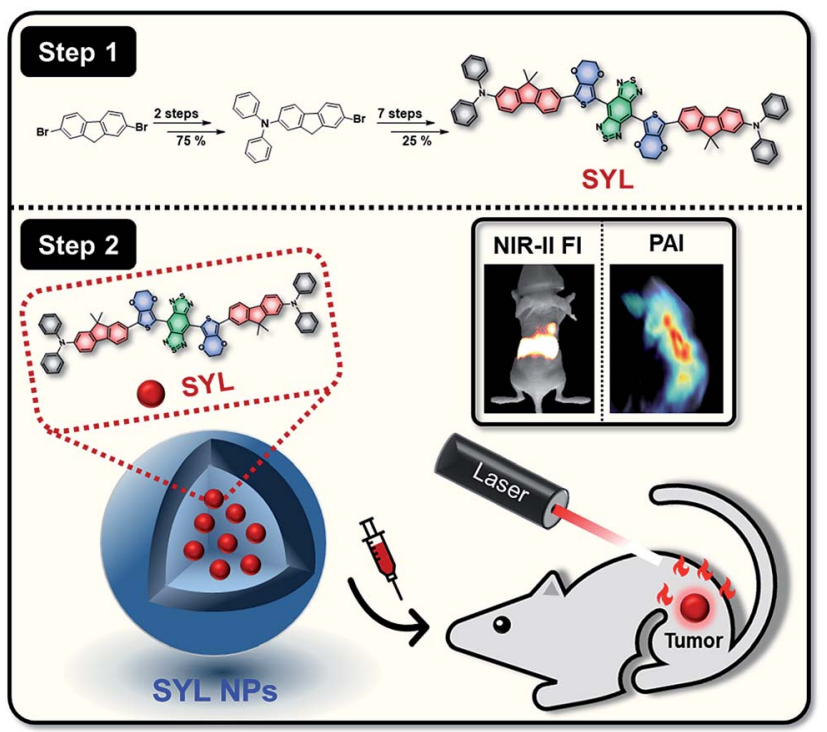

Fig. 1 Schematic illustration of the preparation of SYL NPS, as well as their multifunctional applications in NIR-II/PA dual-modal imaging and photothermal therapy.

electron-density and molar extinction coefficient due to the addition of a secondary electron donor (diphenylamine) to the molecular scaffold. ${ }^{61}$ Moreover, the dialkyl substituted fluorene moiety (first donor) inserted between the second donor and thiophene could extend the $\pi$-conjugated bridge and facilitate intramolecular charge transfer (ICT) (Fig. 1). ${ }^{62}$ 3,4-Ethylene dioxythiophene employed here as the spacer to distort the conjugated backbones could also minimize the intermolecular interactions as well as energy transfer from the molecular excited states to the nearby $\mathrm{H}_{2} \mathrm{O}$ molecules. ${ }^{63}$ The fluorophore SYL with substituted fluorene as the first donor and diphenylamine as the second donor showed the optimized optical performance with strong absorption around $800 \mathrm{~nm}$. This result suggested that under $808 \mathrm{~nm}$ laser irradiation, the optical energy absorbed by SYL could be partly converted into heat to increase the temperature of the surrounding environment and could be utilized to trigger cancer cell death by highly effective and noninvasive photothermal therapy (PTT) ${ }^{64,65}$ In addition, the photothermal effect of SYL generated acoustic waves that could also be detected and converted into photoacoustic (PA) imaging signals. As a hybrid modality, the dual-modal NIR-II/PA imaging meets the requirements of both the superior S/B (signal to background) ratio and high penetration depth for delineating tumor margins, as well as determining the best photothermal treatment time after administration to realize an optimal photothermal effect in cancer therapy. ${ }^{65}$ Therefore, the small molecular dye SYL could effectively integrate dual-modal imaging and photothermal therapeutic functions for cancer theranostics (Fig. 1).

To further endow SYL with excellent solubility and biocompatibility and high preferential passive accumulation at the tumor site, SYL was incorporated into a PEGylated surfactant DSPEmPEG5000 to form nanoparticles (SYL NPs). Based on SYL NPs, in vivo dual-modal NIR-II fluorescence/PA tumor imaging and precise PTT have been performed with excellent results, which could speed up the utilization of small molecular dyes based on D-A-D scaffolds for biomedical applications in near future.

\section{Results and discussion}

A new type of NIR-II small molecular dye, SYL, was designed by using an optimized DD-A-DD scaffold including 3,4-ethylenedioxythiophene and dialkylfluorene and diphenylamine donor units; both contributed to enhancing the fluorescence signals as compared to the previously reported D-A-D dye H1 (Fig. 2) ${ }^{46}$ SYL was synthesized through a convergent route in $25 \%$ overall yield in 9 steps (Fig. 1) and well-characterized by ${ }^{1} \mathrm{H} /{ }^{13} \mathrm{C}-\mathrm{NMR}$ and MALDI-TOF-MS (Fig. S23-S25 $\dagger$ ). Density functional theory (DFT) calculations with the B3LYP (Gaussian 09) functional using the 6-31G basis set were employed for calculating the HOMO and LUMO of both H1 and SYL, respectively. As shown in Fig. 2, the energy bandgap of SYL (1.36 $\mathrm{eV})$ was higher than that of $\mathbf{H 1}(1.17 \mathrm{eV})$.

SYL demonstrated a hypsochromic shift in absorption and emission wavelengths as well as a higher NIR-II fluorescence
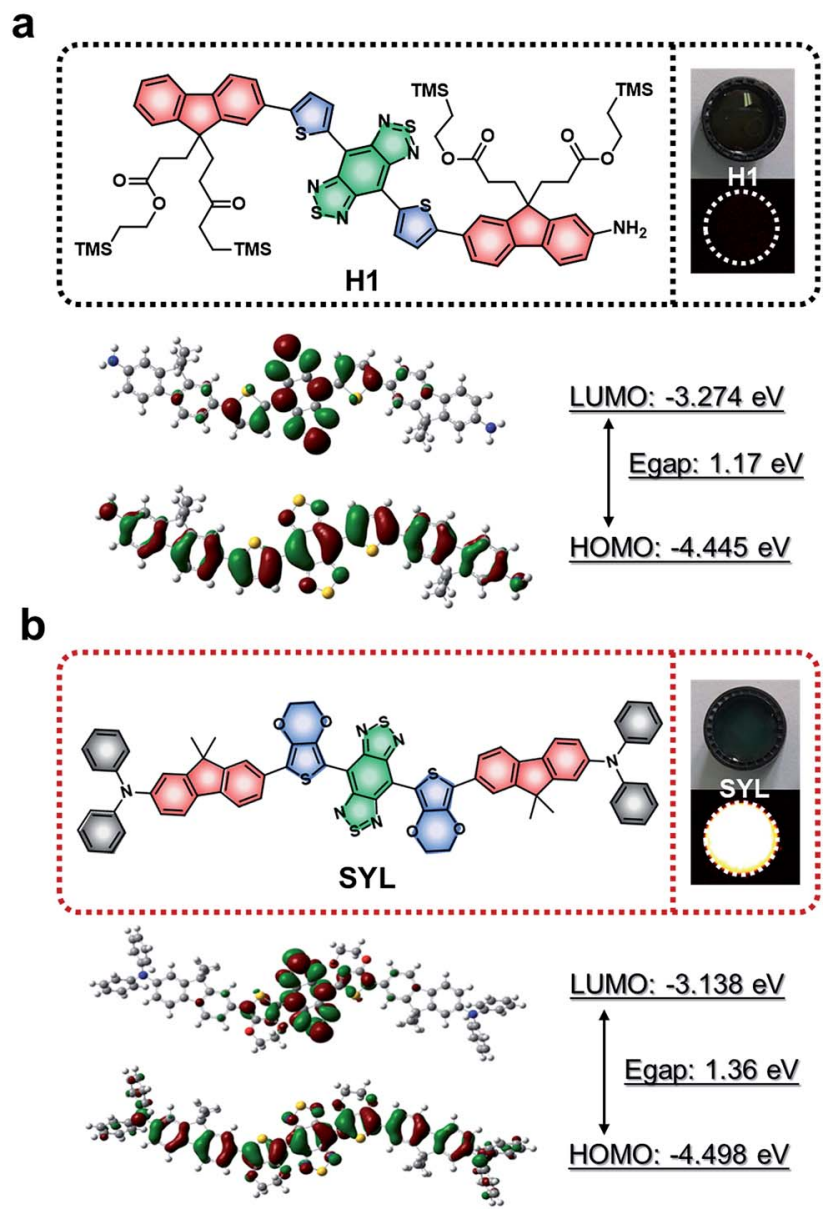

Fig. 2 Chemical structures and electronic properties of SYL and the previously reported NIR-II dye $\mathrm{H} 1$; (a) Frontier orbitals of $\mathrm{H} 1$, its associated energies (DFT using B3LYP Gaussian 09), and NIR-II fluorescence signals. (b) Chemical structures, NIR-II fluorescence signals and Frontier orbitals with associated energies of the novel NIR-II dye SYL. 
signal output compared to H1. To enhance the capability of SYL for in vivo applications, SYL was incorporated into DSPEmPEG5000 (1: 9, w/w) for facile preparation of SYL NPs with $81.6 \pm 2.4 \%$ encapsulation efficiency (Fig. S1 $\dagger$ ). The absorption and emission wavelengths of SYL NPs were detected at $\sim 765 \mathrm{~nm}$ and $\sim 976 \mathrm{~nm}$, respectively (Fig. 3a). Based on transmission electron microscopy (TEM) and dynamic light scattering (DLS) results, the as-synthesized SYL NPs were discrete and uniform with an average size of $\sim 120 \mathrm{~nm}$, suggesting high monodispersity (Fig. 3b). The stability of the SYL NPs was tested in phosphate-buffered saline (PBS) containing 10\% (v/v) fetal bovine serum (FBS) at $37{ }^{\circ} \mathrm{C}$. Even after $48 \mathrm{~h}$, there was no apparent change in the particle-diameter distribution, suggesting the excellent in vitro stability of SYL NPs (Fig. S2 $\dagger$ ). Furthermore, the FDA-approved NIR-I theranostic agent
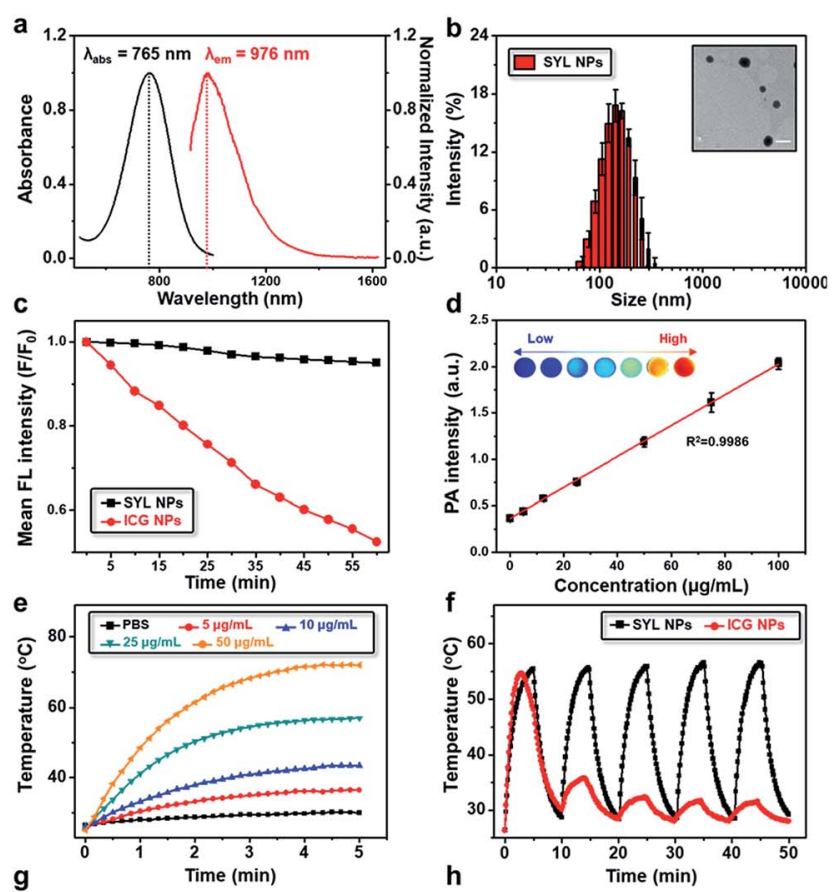

g
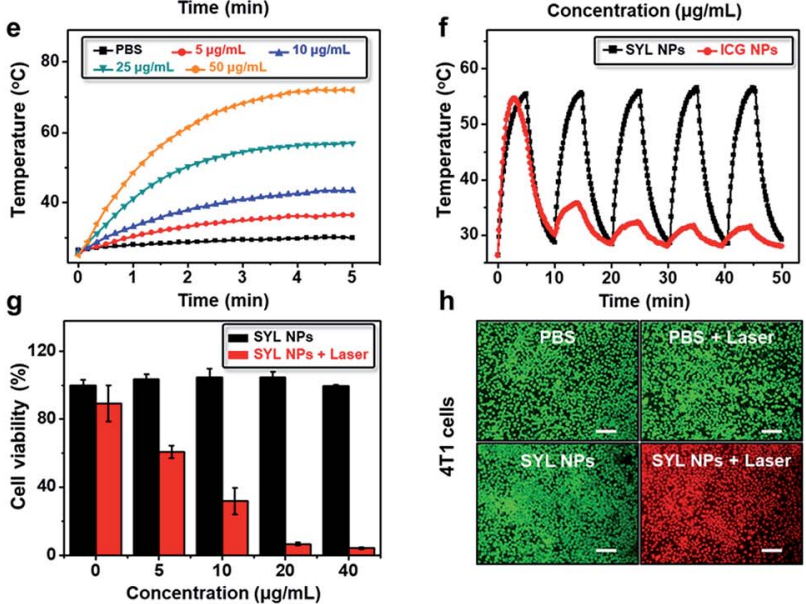

Fig. 3 In vitro NIR-II, PA, photothermal properties and cell viabilities of SYL NPs. (a) UV-Vis-NIR absorption and fluorescence emission of SYL NPs; (b) DLS data and TEM image (inset) of SYL NPs. Scale bar: $200 \mathrm{~nm}$; (c) photostability of SYL NPs and ICG NPs under continuous irradiation at $808 \mathrm{~nm}\left(1 \mathrm{~W} \mathrm{~cm}^{-2}\right.$ ) for $1 \mathrm{~h}$; (d) PA intensity of SYL NPs as a function of concentration from 0 to $100 \mu \mathrm{g} \mathrm{m}$; (e) photothermal conversion behavior of SYL NPs in PBS solution at various concentrations under laser irradiation ( $1 \mathrm{~W} \mathrm{~cm}^{-2}$ ); ( $\mathrm{f}$ ) the photothermal heating and natural cooling cycles of SYL NPs and ICG NPs in PBS solution $\left(25 \mu \mathrm{g} \mathrm{mL}^{-1}, 1\right.$ $\mathrm{W} \mathrm{cm}^{-2}$ ); (g) cell viabilities of 4T1 cancer cells after incubation with SYL NPs with or without laser irradiation; (h) confocal fluorescence images of live (green) and dead (red) 4T1 cells with calcein-AM and PI staining after various treatments. Scale bar: $100 \mu \mathrm{m}$. indocyanine green (ICG) was also incorporated into DSPEPEG5000 to serve as the control group, ICG NPs. SYL NPs exhibited high photo-stability with a negligible decay as compared with ICG NPs under continuous irradiation at 808 $\mathrm{nm}$ for $1 \mathrm{~h}$ (Fig. 3c). Also, SYL in DMSO and SYL NPs in different media did not show any significant change in fluorescence signal intensity upon irradiation $\left(1 \mathrm{~W} \mathrm{~cm}^{-2}\right)$ up to $1 \mathrm{~h}$ (Fig. S3$\mathrm{S} 4 \dagger)$. The PA spectrum showed a broad band extending over a wide NIR-I region (700-900 nm, Fig. S5 $\dagger$ ) and hence SYL NPs could furnish a promising PA signal under $760 \mathrm{~nm}$ irradiation (Fig. S5 $\dagger$ ). Moreover, the PA amplitudes of SYL NPs showed excellent linearity with their molar concentration in a range from 0 to $100 \mu \mathrm{g} \mathrm{mL}^{-1}$ based on SYL (Fig. 3d).

The photothermal properties of SYL NPs were next tested using different power laser irradiations ( 1 to $2 \mathrm{~W} \mathrm{~cm}^{-2}, 808 \mathrm{~nm}$ ). A significant temperature enhancement was observed, suggesting the potential of SYL NPs for photothermal therapy (Fig. S6 $\dagger$ ). In addition, the temperature was found to increase more rapidly with the increased concentrations of SYL NPs (0 to $\left.50 \mu \mathrm{g} \mathrm{mL}^{-1}\right)$ under $808 \mathrm{~nm}$ laser irradiation (power $1 \mathrm{~W} \mathrm{~cm}^{-2}$ ) (Fig. 3e). The temperature of SYL NPs increased from $25.0^{\circ} \mathrm{C}$ to $68.2^{\circ} \mathrm{C}$ within $180 \mathrm{~s}$ of irradiation while there was no significant temperature rise in the control (PBS) group under the same conditions (Fig. 3e). The change in temperature upon laser irradiation was also monitored by using an infrared thermal camera (Fig. S7 $\dagger$ ). SYL NPs exhibited a reversible photothermal heating and natural cooling phenomenon up to five cycles without any significant change in photothermal conversion efficiency, suggesting their good photothermal stability (Fig. 3f). Compared with SYL NPs, ICG NPs were not stable enough under irradiation (Fig. 3f and S8 $\dagger$ ). The photothermal conversion efficiency of SYL and SYL NPs was $\sim 22.3 \%$ and $\sim 21.8 \%$, respectively (Fig. S9-S10 and the equation in the ESI $\dagger$ ), higher than that of ICG $(15.1 \%) .{ }^{66}$

Given the excellent photothermal features, next we evaluated the therapeutic efficacy of SYL NPs in breast cancer 4T1 cells with or without laser irradiation. The therapeutic effect of SYL NPs was examined quantitatively by using CCK8 assay. As shown in Fig. $3 \mathrm{~g}$, more than $90 \%$ of cells were dead upon treatment with $20 \mu \mathrm{g} \mathrm{mL} \mathrm{mL}^{-1}$ SYL NPs under $808 \mathrm{~nm}$ laser irradiation (power $1 \mathrm{~W} \mathrm{~cm}^{-2}$ ). There was only a slightly higher effect on cell death as the concentration of SYL was increased from 20 to $40 \mu \mathrm{g} \mathrm{mL}{ }^{-1}$. In contrast, no obvious cytotoxicity was observed in cells with SYL NPs up to $40 \mu \mathrm{g} \mathrm{mL}^{-1}$ concentration without light irradiation. Further, SYL NP mediated photothermal cytotoxicity was also demonstrated with calcein-AM and PI staining assays (Fig. 3h). The effect of inducing cancer cell death in 4T1 cells with the combination of SYL NPs and laser-irradiation in vitro was much stronger than that of SYL NPs or laser irradiation alone, showing their potential as an efficient phototherapeutic agent in vivo.

Next, we evaluated the fluorescence imaging (NIR-II) performance of SYL NPs in vivo, with ICG NPs as the control. Lymphatic system imaging was performed on living mice (Fig. S11a and $\mathrm{b}_{\dagger} \dagger$ ). Compared to ICG NPs, the lymphatic vessel between the injection site and sentinel lymph node was clearly distinguished and exhibited a high $\mathrm{S} / \mathrm{B}$ ratio after injection of SYL NPs, thus providing fast and precise mapping of the 
lymphatic system by NIR-II fluorescence imaging. In addition, successful imaging of blood vessels was observed from the surrounding background normal tissue after injection of SYL NPs as compared with ICG NPs (Fig. S11c and $d \dagger$ ).

Based on the above results, the efficacy of SYL NPs for passive targeting and dual-modal imaging of $4 \mathrm{~T} 1$ tumors in vivo was further investigated. After the 4T1 tumor-bearing nude mice $(n=$ 3) were intravenously injected with $100 \mu \mathrm{g}$ of SYL NPs in PBS, NIR-II fluorescence images (Series III 900/1700 equipment, NIROptics Tech. Co. Ltd., Suzhou) and PA signals of living mice were constantly monitored. From both NIR-II fluorescence and PA images at 2, 8, 12 and $24 \mathrm{~h}$ post-injection, the tumor was clearly visualized from the surrounding background tissues and showed passive uptake of SYL NPs at all time points (Fig. 4a). The semiquantitative analysis of both NIR-II and PA images further revealed that the tumor uptake of SYL NPs gradually increased with time and reached a maximum at $8 \mathrm{~h}$ (Fig. 4b). Thereafter, the signal from the localized SYL NPs at the tumor region site reduced gradually during $8-24 \mathrm{~h}$. As seen in Fig. $4 \mathrm{c}$, the S/B ratio of both NIR-II and PA imaging increased steadily in the first $2 \mathrm{~h}$ and reached a maximum value at $8 \mathrm{~h}$ post-injection. To further confirm this, ex vivo imaging of tumors and other major organs/ tissues was performed $24 \mathrm{~h}$ post-injection. The NIR-II fluorescence signals in the excised tumor, liver and spleen were much brighter than those of other organs (Fig. S12†). These findings were consistent with the in vivo imaging results, demonstrating that SYL NPs possessed favorable in vivo distribution, higher tumor accumulation, and hepatobiliary clearance.

Guided by the excellent NIR-II fluorescence/PA imaging both in vitro and in vivo, the PTT efficacy of SYL NPs in mice was then investigated with PBS, PBS with $808 \mathrm{~nm}$ NIR laser irradiation and SYL NPs as negative controls. PBS $(100 \mu \mathrm{L})$ and SYL NPS $(100 \mu \mathrm{g} / 100 \mu \mathrm{L})$ were injected into $4 \mathrm{~T} 1$ tumor-bearing nude mice respectively via the tail vein. As the maximum accumulation of

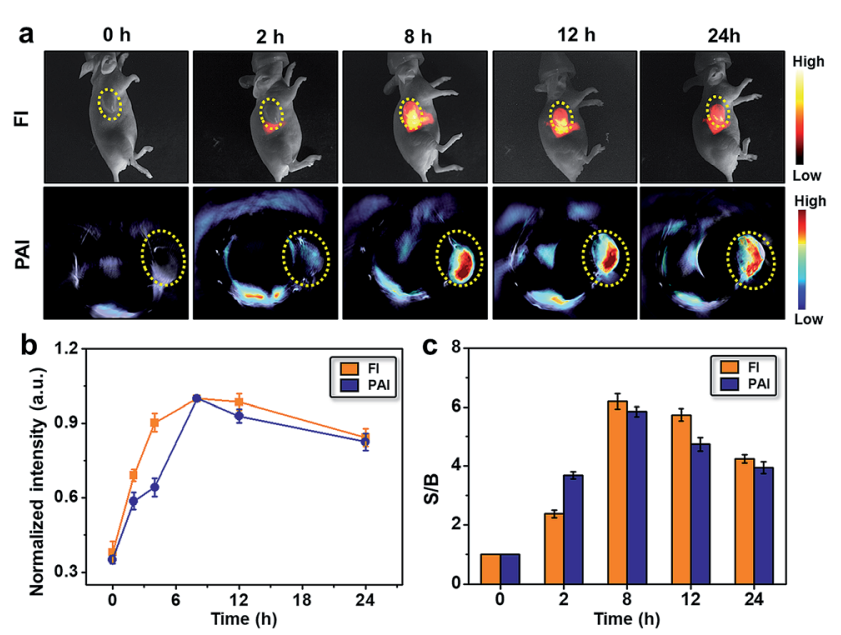

Fig. 4 In vivo multimodal images with SYL NPs. (a) In vivo NIR-II fluorescence (100 ms, LP1000, $82 \mathrm{~mW} \mathrm{~cm}^{-2}$ ) and PA images of 4T1 tumor-bearing mice at $0,2,8,12$, and $24 \mathrm{~h}$ after tail vein injection of SYL NPs. Yellow circles indicate tumor sites; (b) semi-quantified fluorescence and PA intensities; (c) time-dependence of the signal-tobackground ratio (FI \& PA) after intravenous injection.
SYL NPs was around $8 \mathrm{~h}$ according to the in vivo imaging data, PTT treatment was performed at $8 \mathrm{~h}$ post-injection. As seen in Fig. $5 \mathrm{a}-\mathrm{c}$, after $120 \mathrm{~s}$ of irradiation ( $808 \mathrm{~nm}$, power $1 \mathrm{~W} \mathrm{~cm} \mathrm{~cm}^{-2}$ ), the temperature at the tumor site treated with SYL NPs increased to $50.1^{\circ} \mathrm{C}$, which was much higher than that of other controls, i.e., the PBS with $808 \mathrm{~nm}$ NIR laser irradiation group $\left(38.6{ }^{\circ} \mathrm{C}\right)$ (Fig. 5c). Body weight and tumor growth were recorded every other day until 19 days. No significant body weight loss was observed in all the tested groups after treatment (Fig. 5d). Moreover, the result of hematoxylin and eosin (H\&E) stains of major organs excised at the $20^{\text {th }}$ day indicated that SYL NP based PTT was biocompatible without obvious adverse effects (Fig. S13†). Only the tumor growth in the mice treated with both SYL NPs and $808 \mathrm{~nm}$ laser irradiation was obviously inhibited, whereas the growth of the tumors in the other three tested groups exhibited a likewise rapid growth trend (Fig. 5e). Additionally, the survival rate of the 4T1 tumor-bearing mice treated with SYL NPS and laser irradiation was higher than those of the other tested groups after 40 days (Fig. 5f), indicating the effective photothermal properties of SYL NPs. Further, H\&E stains of dissected tumor tissues from the tested groups indicated significant tumor cell damage with nuclear membrane fragmentation and nucleus pyknosis in the SYL NP treated group under laser irradiation (Fig. S14 $\dagger$ ). For the other tested groups, there was no obvious a
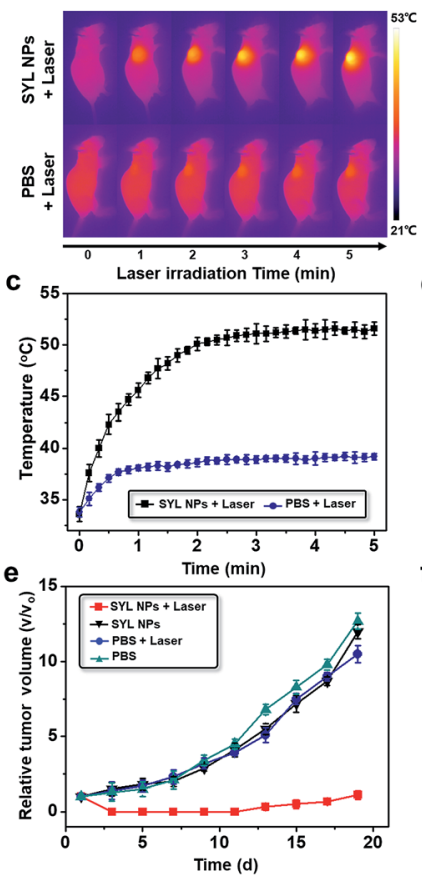

b SYLNPS
+ SYLNPS
+Laser PBS
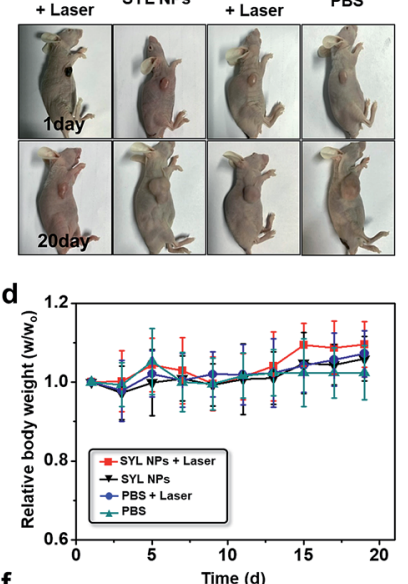

f

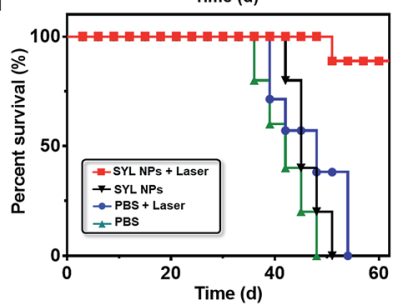

Fig. 5 Therapeutic effect of SYL NPs on tumor-bearing mice via tail vein injection. (a) IR thermal images of $4 \mathrm{~T}-1$ bearing mice injected with SYL NPS and PBS under irradiation ( $\left.808 \mathrm{~nm}, 1 \mathrm{~W} \mathrm{~cm}^{-2}\right)$; (b) images of tumor-bearing mice 20 days post various treatments; (c) temperature changes in the subcutaneous tumor sites of mice treated with SYL NPS and PBS under continuous laser irradiation; (d) analysis of average body weights; (e) tumor growth inhibition curves for PBS alone, PBS \& $808 \mathrm{~nm}$ laser irradiation, SYL NPs alone, and SYL NPs \& $808 \mathrm{~nm}$ laser irradiation; (f) survival curves of the $4 \mathrm{~T} 1$ tumor-bearing mice groups after different treatments. 
tumor cell damage. The results suggested that SYL NPs under laser irradiation displayed high in vivo photothermal therapeutic efficiency in the 4T1 tumor-bearing model.

\section{Conclusions}

In summary, a novel DD-A-DD scaffold molecular dye SYL was designed and facilely synthesized with intrinsic multifunctional ability including dual-modal NIR-II fluorescence/PA imaging as well as photothermal therapeutic effects. The SYL NP based probe has the capability to passively target tumors in the 4T1 subcutaneous mouse model with relatively high fluorescence and photoacoustic signal outputs as well as an excellent S/B ratio. Additionally, the efficient tumor inhibition rate with minimal adverse side effects prolonged the life span of tumorbearing mice in the SYL NP treated group under laser irradiation. These results highlighted that SYL NPs as a multifunctional platform could be an interesting candidate for cancer diagnosis (NIR-II) and therapy in future bioapplications. However, stimulated by hyperthermia at the tumor site, incompletely killed tumor cells might have chances of recurrence because of thermal resistance acquired by these residual tumor cells. ${ }^{67}$ To improve PTT efficiency, some suggestions are recommended here. First, dyes with a longer emission wavelength and higher photothermal conversion efficiency should be designed, which can ensure enough penetration depth to cover the whole tumor volume and sufficient temperature upon irradiation to kill the cancer cells completely. Second, integrating PTT with other therapeutic modalities such as photodynamic therapy (PDT), chemotherapy or gene therapy could be beneficial to improve the overall treatment efficiency.

\section{Ethical statement}

The facilities used for animal studies have been accredited by the Association for Assessment and Accreditation of Laboratory Animal Care International (AAALAC International), and all subsequent procedures were approved by and performed in accordance with the Guidelines for the Care and Use of Laboratory Animals of the Chinese Animal Welfare Committee.

\section{Conflicts of interest}

There are no conflicts to declare.

\section{Acknowledgements}

This work was partially supported by grants from the National Key R\&D Programgs1: (2017YFA0505203), NSFC (21708012), 111 project B17019, and NSFHP (2017CFB151) and financially supported by self-determined research funds of CCNU from the colleges (1100-30106190234), basic research and operation of MOE, Wuhan Morning Light Plan of Youth Science and Technology (201705304010321), Wuhan scientific and technological projects (2019020701011441), the Open Research Fund of Jiangsu Key Laboratory of Medical Optics (JKLMO201803) from Jiangsu Key Laboratory of Medical Optics, Suzhou Institute of
Biomedical Engineering and Technology, and the Chinese Academy of Sciences, Suzhou 215163 (P. R. China). We are also thankful to research grants numbered 2018R1A3B1052702 and NRF-2019M3E5D1A01068998 (J. S. K.). Thanks to Suzhou NIROptics Technologies Co., Ltd (Suzhou, China). Without their technical help, this project could not have proceeded smoothly.

\section{Notes and references}

1 Y. Cai, Z. Wei, C. Song, C. Tang, W. Han and X. Dong, Chem. Soc. Rev., 2019, 48, 22-37.

2 J. Li and K. Pu, Chem. Soc. Rev., 2019, 48, 38-71.

3 M. C. Heffern, L. M. Matosziuk and T. J. Meade, Chem. Rev., 2014, 114, 4496-4539.

4 S. He, J. Song, J. Qu and Z. Cheng, Chem. Soc. Rev., 2018, 47, 4258-4278.

5 X. Huang, J. Song, B. C. Yung, X. Huang, Y. Xiong and X. Chen, Chem. Soc. Rev., 2018, 47, 2873-2920.

6 M. H. Lee, A. Sharma, M. J. Chang, J. Lee, S. Son, J. L. Sessler, C. Kang and J. S. Kim, Chem. Soc. Rev., 2018, 47, 28-52.

7 L. He, B. Dong, Y. Liu and W. Lin, Chem. Soc. Rev., 2016, 45, 6449-6461.

8 Y. Xing, J. Zhao, P. S. Conti and K. Chen, Theranostics, 2014, 4, 290-306.

9 A. Louie, Chem. Rev., 2010, 110, 3146-3195.

10 Y. Sun, X. Ma, K. Cheng, B. Wu, J. Duan, H. Chen, L. Bu, R. Zhang, X. Hu, Z. Deng, L. Xing, X. Hong and Z. Cheng, Angew. Chem., Int. Ed., 2015, 54, 5981-5984.

11 H. S. Jung, P. Verwilst, A. Sharma, J. Shin, J. L. Sessler and J. S. Kim, Chem. Soc. Rev., 2018, 47, 2280-2297.

12 J. Ma, M. Yu, Z. Zhang, W. Cai, Z. Zhang, H. Zhu, Q. Cheng, Z. Tian and D. Pang, Nanoscale, 2018, 10, 10699-10704.

13 R. Huang, M. Wang, Y. Zhu, P. S. Conti and K. Chen, Curr. Top. Med. Chem., 2015, 15, 795-819.

14 Y. Sun, X. Zeng, Y. Xiao, C. Liu, H. Zhu, H. Zhou, Z. Chen, F. Xu, J. Wang, M. Zhu, J. Wu, H. Zhang, Z. Deng, Z. Cheng and X. Hong, Chem. Sci., 2018, 9, 2092-2097.

15 L. Sun, J. Ding, W. Xing, Y. Gai, J. Sheng and D. Zeng, Bioconjugate Chem., 2016, 27, 1200-1204.

16 Y. Gai, G. Xiang, X. Ma, W. Hui, Q. Ouyang, L. Sun, J. Ding, J. Sheng and D. Zeng, Bioconjugate Chem., 2016, 27, 515-520.

17 H. Zhang, P. Desai, Y. Koike, J. Houghton, S. Carlin, N. Tandon, K. Touijer and W. A. Weber, J. Nucl. Med., 2017, 58, 29-35.

18 F. Ding, Y. Zhan, X. Lu and Y. Sun, Chem. Sci., 2018, 9, 43704380.

19 F. Ding, S. Chen, W. Zhang, Y. Tu and Y. Sun, Bioorg. Med. Chem., 2017, 25, 5179-5184.

20 G. Hong, A. L. Antaris and H. Dai, Nat. Biomed. Eng., 2017, 1, 0010.

21 Y. Fan, P. Wang, Y. Lu, R. Wang, L. Zhou, X. Zheng, X. Li, J. A. Piper and F. Zhang, Nat. Nanotechnol., 2018, 13, 941-946. 22 Q. Miao and K. Pu, Adv. Mater., 2018, 30, 1801778.

23 Kenry, Y. Duan and B. Liu, Adv. Mater., 2018, 30, 1802394.

24 C. Li and Q. Wang, ACS Nano, 2018, 12, 9654-9659.

25 Y. Fan and F. Zhang, Adv. Opt. Mater., 2019, 7, 1801417.

26 Z. Xue, S. Zeng and J. Hao, Biomaterials, 2018, 171, 153-163. 
27 R. Wang, L. Zhou, W. Wang, X. Li and F. Zhang, Nat. Commun., 2017, 8, 14702.

28 S. Zhu, B. C. Yung, S. Chandra, G. Niu, A. L. Antaris and X. Chen, Theranostics, 2018, 8, 4141-4151.

29 Y. Tang, Y. Li, X. Lu, X. Hu, H. Zhao, W. Hu, F. Lu, Q. Fan and W. Huang, Adv. Funct. Mater., 2019, 29, 1807376.

30 Y. Tang, Y. Li, X. Hu, H. Zhao, Y. Ji, L. Chen, W. Hu, W. Zhang, X. Li, X. Lu, W. Huang and Q. Fan, Adv. Mater., 2018, 30, 1801140.

31 X. Lei, R. Li, D. Tu, X. Shang, Y. Liu, W. You, C. Sun, F. Zhang and X. Chen, Chem. Sci., 2018, 9, 4682-4688.

32 C. Ruan, C. Liu, H. Hu, X. L. Guo, B. P. Jiang, H. Liang and X. C. Shen, Chem. Sci., 2019, 10, 4699-4706.

33 P. Sun, Q. Wu, X. Sun, H. Mao, W. Deng, W. Zhang, Q. Fan and W. Huang, Chem. Commun., 2018, 54, 13395-13398.

34 Y. Tang, Y. Li, Z. Wang, F. Pei, X. Hu, Y. Ji, X. Li, H. Zhao, W. Hu, X. Lu, Q. Fan and W. Huang, Chem. Commun., 2019, 55, 27-30.

35 F. Ding, Y. Fan, Y. Sun and F. Zhang, Adv. Healthcare Mater., 2019, 8, 1900260.

36 B. Li, L. Lu, M. Zhao, Z. Lei and F. Zhang, Angew. Chem., Int. Ed., 2018, 57, 7483-7487.

37 L. Liu, S. Wang, B. Zhao, P. Pei, Y. Fan, X. Li and F. Zhang, Angew. Chem., Int. Ed., 2018, 57, 7518-7522.

38 G. Xu, Q. Yan, X. Lv, Y. Zhu, K. Xin, B. Shi, R. Wang, J. Chen, W. Gao, P. Shi, C. Fan, C. Zhao and H. Tian, Angew. Chem., Int. Ed., 2018, 57, 3626-3630.

39 A. L. Antaris, H. Chen, K. Cheng, Y. Sun, G. Hong, C. Qu, S. Diao, Z. Deng, X. Hu, B. Zhang, X. Zhang, O. K. Yaghi, Z. R. Alamparambil, X. Hong, Z. Cheng and H. Dai, Nat. Mater., 2016, 15, 235-242.

40 A. L. Antaris, H. Chen, S. Diao, Z. Ma, Z. Zhang, S. Zhu, J. Wang, A. X. Lozano, Q. Fan, L. Chew, M. Zhu, K. Cheng, X. Hong, H. Dai and Z. Cheng, Nat. Commun., 2017, 8, 15269.

41 Y. Sun, C. Qu, H. Chen, M. He, C. Tang, K. Shou, S. Hong, M. Yang, Y. Jiang, B. Ding, Y. Xiao, L. Xing, X. Hong and Z. Cheng, Chem. Sci., 2016, 7, 6203-6207.

42 F. Ding, C. Li, Y. Xu, J. Li, H. Li, G. Yang and Y. Sun, Adv. Healthcare Mater., 2018, 7, 1800973.

43 Q. Yang, Z. Hu, S. Zhu, R. Ma, H. Ma, R. Ma, H. Wan, T. Zhu, Z. Jiang, W. Liu, L. Jiao, H. Sun, Y. Liang and H. Dai, J. Am. Chem. Soc., 2018, 140, 1715-1724.

44 S. Zhu, Q. Yang, A. L. Antaris, J. Yue, Z. Ma, H. Wang, W. Huang, H. Wan, J. Wang, S. Diao, B. Zhang, X. Li, Y. Zhong, K. Yu, G. Hong, J. Luo, Y. Liang and H. Dai, Proc. Natl. Acad. Sci. U. S. A., 2017, 114, 962-967.

45 K. Shou, C. Qu, Y. Sun, H. Chen, S. Chen, L. Zhang, H. Xu, X. Hong, A. Yu and Z. Cheng, Adv. Funct. Mater., 2017, 27, 1700995.

46 Y. Sun, M. Ding, X. Zeng, Y. Xiao, H. Wu, H. Zhou, B. Ding, C. Qu, W. Hou, A. Er-bu, Y. Zhang, Z. Cheng and X. Hong, Chem. Sci., 2017, 8, 3489-3493.

47 L. Tu, Y. Xu, Q. Ouyang, X. Li and Y. Sun, Chin. Chem. Lett., 2019, DOI: 10.1016/j.cclet.2019.05.022.

48 Y. Sun, F. Ding, Z. Zhou, C. Li, M. Pu, Y. Xu, Y. Zhan, X. Lu, H. Li, G. Yang, Y. Sun and P. J. Stang, Proc. Natl. Acad. Sci. U. S. A., 2019, 116, 1968-1973.
49 X. Zhang, H. Wang, A. L. Antaris, L. Li, S. Diao, R. Ma, A. Nguyen, G. Hong, Z. Ma, J. Wang, S. Zhu, J. M. Castellano, T. Wyss-Coray, Y. Liang, J. Luo and H. Dai, Adv. Mater., 2016, 28, 6872-6879.

50 Q. Yang, Z. Ma, H. Wang, B. Zhou, S. Zhu, Y. Zhong, J. Wang, H. Wan, A. L. Antaris, R. Ma, X. Zhang, J. Yang, X. Zhang, H. Sun, W. Liu, Y. Liang and H. Dai, Adv. Mater., 2017, 29, 1605497.

51 H. Wan, J. Yue, S. Zhu, T. Uno, X. Zhang, Q. Yang, K. Yu, G. Hong, J. Wang, L. Li, Z. Ma, H. Gao, Y. Zhong, J. Su, A. L. Antaris, Y. Xia, J. Luo, Y. Liang and H. Dai, Nat. Commun., 2018, 9, 1171-1179.

52 S. Zhu, S. Herraiz, J. Yue, M. Zhang, H. Wan, Q. Yang, Z. Ma, Y. Wang, J. He, A. L. Antaris, Y. Zhong, S. Diao, Y. Feng, Y. Zhou, K. Yu, G. Hong, Y. Liang, A. J. Hsueh and H. Dai, Adv. Mater., 2018, 30, 1705799.

53 X. Zeng, Y. Xiao, J. Lin, S. Li, J. Nong, G. Xu, H. Wang, F. Xu, J. Wu, Z. Deng and X. Hong, Adv. Healthcare Mater., 2018, 7, 1800589.

54 J. Lin, X. Zeng, Y. Xiao, L. Tang, J. Nong, Y. Liu, H. Zhou, B. Ding, F. Xu, H. Tong, Z. Deng and X. Hong, Chem. Sci., 2019, 10, 1219-1226.

55 R. Tian, H. Ma, Q. Yang, H. Wan, S. Zhu, S. Chandra, H. Sun, D. O. Kiesewetter, G. Niu, Y. Liang and X. Chen, Chem. Sci., 2019, 10, 326-332.

56 K. Cheng, H. Chen, C. H. Jenkins, G. L. Zhang, W. Zhao, Z. Zhang, F. Han, J. Fung, M. Yang, Y. Jiang, L. Xing and Z. Cheng, ACS Nano, 2017, 11, 12276-12291.

57 Y. Yuan, C. Zhang, M. Gao, R. Zhang, B. Tang and B. Liu, Angew. Chem., Int. Ed., 2015, 54, 1780-1786.

58 J. Qi, C. Sun, A. Zebibula, H. Zhang, R. K. Kwok, X. Zhao, W. Xi, J. W. Y. Lam, J. Qian and B. Tang, Adv. Mater., 2018, 30, 1706856.

59 Z. Sheng, B. Guo, D. Hu, S. Xu, W. Wu, W. H. Liew, K. Yao, J. Jiang, C. Liu, H. Zheng and B. Liu, Adv. Mater., 2018, 30, 1800766.

60 J. Qi, C. Sun, D. Li, H. Zhang, W. Yu. A. Zebibula, J. W. Y. Lam, W. Xi, L. Zhu, F. Cai, P. Wei, C. Zhu, R. T. K. Kwok, L. L. Streich, R. Prevedel, J. Qian and B. Z. Tang, ACS Nano, 2018, 12, 7936-7945.

61 K. R. J. Thomas, Y. C. Hsu, J. T. Lin, K. M. Lee, K. C. Ho, C. H. Lai, Y. M. Cheng and P. T. Chou, Chem. Mater., 2008, 20, 1830-1840.

62 P. Shen, Y. Tang, S. Jiang, H. Chen, X. Zheng, X. Wang, B. Zhao and S. Tan, Org. Electron., 2011, 12, 125-135.

63 G. Qian and Z. Wang, Chem.-Asian J., 2010, 5, 1006-1029.

64 Q. Chen, J. Wen, H. Li, Y. Xu, F. Liu and S. Sun, Biomaterials, 2016, 106, 144-166.

65 Y. Liu, P. Bhattarai, Z. Dai and X. Chen, Chem. Soc. Rev., 2019, 48, 2053-2108.

66 M. Zheng, P. Zhao, Z. Luo, P. Gong, C. Zheng, P. Zhang, C. Yue, D. Gao, Y. Ma and L. Cai, ACS Appl. Mater. Interfaces, 2014, 6, 6709-6716.

67 Q. Wang, Y. Dai, J. Xu, J. Cai, X. Niu, L. Zhang, R. Chen, Q. Shen, W. Huang and Q. Fan, Adv. Funct. Mater., 2019, DOI: $10.1002 /$ adfm.201901480. 